USM-TH-117

\title{
$q \rightarrow \Lambda$ Fragmentation Function and Nucleon Transversity Distribution in a Diquark Model
}

\author{
Jian-Jun Yang用 $a, b$ \\ ${ }^{a}$ Department of Physics, Nanjing Normal University, \\ Nanjing 210097, China \\ ${ }^{b}$ Departamento de Física, Universidad Técnica Federico Santa María, \\ Casilla 110-V, Valparaíso, Chilef
}

\begin{abstract}
Based on a simple quark-diquark model, we propose a set of unpolarized, longitudinally polarized and transversely polarized fragmentation functions for the $\Lambda$ by fitting the unpolarized $\Lambda$ production data in $e^{+} e^{-}$annihilation. It is found that the helicity structure of the obtained $\Lambda$ fragmentation functions is supported by the all available experimental data on the longitudinal $\Lambda$ polarization. Within the same framework of the diquark model, the nucleon transversity distributions are presented and consistent descriptions of the available HERMES data on the azimuthal spin asymmetries in pion electroproduction are obtained. Furthermore, the spin transfers to the transversely polarized $\Lambda$ in the charged lepton DIS on a transversely polarized nucleon target are predicted for future experiments.
\end{abstract}

(Talk given in a seminar of U. Santa María)

*e-mail: jjyang@fis.utfsm.cl

${ }^{\dagger}$ Mailing address 


\section{Introduction}

In recent years, there has been great progress in understanding the detailed flavor and spin structure of the nucleon. In order to enrich our knowledge of the nucleon structure, it is significant to apply the same mechanisms that produce the quark structure of the nucleon to other octet baryons and to find a new domain where the physics invoked to explain the structure of the nucleon can be clearly checked. The $\Lambda$ hyperon is of special interest in this respect since its polarization can be easily measured in the usual fashion through the self-analyzing decay $\Lambda \rightarrow p \pi^{-}$. Actually, there has been some recent progress in measurements of polarized $\Lambda$ production. The longitudinal $\Lambda$ polarization in $e^{+} e^{-}$annihilation at the Z-pole was observed by several collaborations at CERN [1-3]. Recently, the HERMES Collaboration at DESY reported a result for the longitudinal spin transfer to the $\Lambda$ in the polarized positron deep inelastic scattering (DIS) process 四. Also the E665 Collaboration at FNAL measured the $\Lambda$ and $\bar{\Lambda}$ spin transfers from muon DIS [5], and they observed a very different behaviour for $\Lambda$ 's and $\bar{\Lambda}$ 's. Very recently, the measurement of $\Lambda$ polarization in charged current interactions has been done in the NOMAD experiment [6]. On the other hand, much work has already been done to relate the flavor and spin structure of the $\Lambda$ to various fragmentation processes [7-25]. One of the most interesting observations is related to the polarization of quarks inside the $\Lambda$. In the naive quark model, the $\Lambda$ spin is exclusively provided by the strange (s) quark, and the $u$ and $d$ quarks are unpolarized. Based on novel results concerning the proton spin structure from DIS experiments and $\mathrm{SU}(3)$ symmetry in the baryon octet, it was found that the $u$ and $d$ quarks of the $\Lambda$ should be negatively polarized [ $[\pi$. However, based on the light cone $\mathrm{SU}(6)$ quark diquark spectator model and the perturbative QCD (pQCD) counting rules analysis, it was found that the $u$ and $d$ quarks should be positively polarized at large $x$, even though their net spin contributions to the $\Lambda$ might be zero or negative [13]. Very recent analysis [14] with a statistical model also supports the above observation. In consideration of the fact that what one can actually measure in experiments are the quark to $\Lambda$ fragmentation functions, some models for the fragmentation functions have been proposed [10]. The unpolarized and polarized 
fragmentation functions were modeled but lack a close connection to the experimental data. Along another direction, the unpolarized $\Lambda$ fragmentation functions have been parametrized [15] with the assumption of $\mathrm{SU}(3)$ flavor symmetry and various sets of polarized $\Lambda$ fragmentation functions have been proposed [15, 17, 21] based on a simple ansatz such as $\Delta D_{q}^{\Lambda}(z)=C_{q}(z) D_{q}^{\Lambda}(z)$ with some assumed coefficients $C_{q}(z)$, or Monte Carlo event generators without a clear physics motivation. The high statistics investigation of polarized $\Lambda$ production is one of the main future goals of the HERMES Collaboration which will improve their detector for this purpose by adding so called Lambda-wheels. It is very timely as more reliable unpolarized and polarized $\Lambda$ fragmentation functions with a clear physics motivation are necessary to serve as basis for the analysis of the new HERMES data.

Another significant approach of enriching our knowledge of the nucleon structure is to extend the analysis from the unpolarized and helicity distributions to the transverse distributions of the nucleon. The unpolarized, helicity and transverse distributions are three fundamental quark distributions of the nucleon. The unpolarized and helicity distributions have been known with some precision both experimentally and theoretically. The quark transverse distributions of the nucleon are less known since they are not directly observables in inclusive DIS processes. Among the proposals to measure the quark transverse distributions, the azimuthal asymmetry in semi-inclusive hadron production has been considered [26-30] through the Collins effect [26] of non-zero production between a chiral-odd structure function and a T-odd fragmentation function. Actually, the HERMES Collaboration [31] has recently reported evidence for single-spin asymmetries for semi-inclusive pion production in deep inelastic scattering of unpolarized positron beam on the longitudinally polarized proton target. Significant spin asymmetries of the distribution in azimuthal angle $\phi$ of the $\pi^{+}$and $\pi^{0}$ relative to the lepton scattering plane have been observed. It is found that the azimuthal asymmetries can provide information on the quark transverse distributions of the nucleon. In addition, Artru and Mekhfi [33], and later Jaffe [16], have noticed that the $\Lambda$-hyperon transverse polarization, in the current fragmentation region of charged lepton DIS on the transversely polarized nucleon target, can also provide information on the quark transverse distributions of the target. Thus 
it is possible to make a systematic study on the quark transverse distributions of the nucleon and the polarized $q \rightarrow \Lambda$ fragmentation functions by using the available facilities, such as COMPASS, HERMES and SMC, for detecting $\Lambda$ fragmentation in charged lepton DIS on both longitudinally and transversely polarized nucleon targets. In fact there is a real need for more realistic predictions for the quark transverse distributions of the nucleon for future experiments.

We have noticed that a simple quark diquark model given in Ref. [10] with a clear physics picture can be used to provide realistic predictions for the $\Lambda$ fragmentation functions and the quark transverse distributions of the nucleon including the $\mathrm{SU}(3)$ symmetry breaking effect. We plan to propose a set of unpolarized, longitudinally polarized and transversely polarized $q \rightarrow \Lambda$ fragmentation functions based on the quark-diquark model [10]. The unpolarized $\Lambda$ fragmentation functions are optimized by a fit to the unpolarized cross section of the produced $\Lambda$ in $e^{+} e^{-}$annihilation. Then we relate the polarized $\Lambda$ fragmentation functions to the unpolarized ones at the initial scale by using some inputs from the diquark model calculation and check the obtained fragmentation functions by means of the available measurement results on the $\Lambda$ polarization. Within the same framework of the diquark model, the quark transverse distributions of the nucleon will also be provided. Furthermore, we predict the azimuthal spin asymmetry in pion electroproduction and the spin transfer to the transversely polarized $\Lambda$ in the charged lepton DIS on a transversely polarized nucleon target.

This paper is a simply extension of our previous work [11] with a purpose of checking the transversely polarized fragmentation functions and nucleon transversity. In Sec. 2, we describe the quark to $\Lambda$ fragmentation functions based on the quarkdiquark model. In Sec. 3, the comparisons between our predictions of the longitudinal polarization for the $\Lambda$ produced in various processes and the corresponding available experimental data are made. In Sec. 4, the quark transverse distributions of the nucleon are proposed based on the diquark model. In Sec. 5, the calculated results of the azimuthal spin asymmetries in pion electroproduction are shown to be consistent with the available HERMES data. In Sec. 6, we predict the spin transfer to the transversely polarized $\Lambda$ in the charged lepton DIS on a transversely polarized nucleon 
target. Finally, we give a brief discussion and summary with our conclusions in Sec. 7.

\section{$2 \quad q \rightarrow \Lambda$ fragmentation functions}

Theoretically, the quark fragmentation functions appear in the QCD description of hard processes as the parts that connect the quark and gluon lines to hadrons in the final state. Following Ref. [10], the fragmentation of a quark into a specific hadron $(p, \Lambda, \ldots)$ can be modeled with a quark-diquark-hadron vertex and the form factors for scalar and axial vector diquark are taken as the same form

$$
\phi\left(k^{2}\right)=N \frac{k^{2}-m_{q}^{2}}{\left(k^{2}-\Lambda_{0}^{2}\right)^{2}}
$$

with a normalization constant $N$ and a mass parameter $\Lambda_{0} . \Lambda_{0}=500 \mathrm{MeV}$ will be adopted in our numerical calculations. In Eq. (四), $m_{q}$ and $k$ are the mass and the momentum of the fragmenting quark $q$, respectively.

Within the framework of the diquark model [10, the unpolarized valence quark to $\Lambda$ fragmentation functions can be expressed as

$$
\begin{gathered}
D_{u_{v}}^{\Lambda}(z)=D_{d_{v}}^{\Lambda}(z)=\frac{1}{12} a_{S}^{(u)}(z)+\frac{1}{4} a_{V}^{(u)}(z), \\
D_{s_{v}}^{\Lambda}(z)=\frac{1}{3} a_{S}^{(s)}(z),
\end{gathered}
$$

where $a_{D}^{(q)}(z)(D=S$ or $V)$ is the probability of finding a quark $q$ splitting into $\Lambda$ with longitudinal momentum fraction $z$ and emitting a scalar $(S)$ or axial vector $(V)$ antidiquark. The SU(3) symmetry breaking effect can be reflected by considering the mass difference between the scalar diquark and vector diquark states.

According to Ref. [10], $a_{D}^{(q)}(z)$ can be expressed in the quark-diquark model as

$$
a_{D}^{(q)}(z)=\frac{N^{2} z^{2}(1-z)^{3}}{64 \pi^{2}} \frac{\left[2\left(M_{\Lambda}+m_{q} z\right)^{2}+R^{2}(z)\right]}{R^{6}(z)}
$$

with

$$
R(z)=\sqrt{z m_{D}^{2}-z(1-z) \Lambda_{0}^{2}+(1-z) M_{\Lambda}^{2}},
$$


where $M_{\Lambda}$ and $m_{D}(D=S$ or $V)$ are the mass of the $\Lambda$ and a diquark, respectively. In consideration of the mass difference $M_{\Lambda}-M_{p}=176 \mathrm{MeV}$, we choose the diquark mass $m_{S}=900 \mathrm{MeV}$ and $m_{V}=1100 \mathrm{MeV}$ for non-strange diquark states, $m_{S}=$ $(900+176) \mathrm{MeV}$ and $m_{V}=(1100+176) \mathrm{MeV}$ for diquark states $(q s)$ with $q=u, d$. The quark masses are taken as $m_{u}=m_{d}=350 \mathrm{MeV}$ and $m_{s}=(350+176) \mathrm{MeV}$.

Similarly, the longitudinally and transversely polarized quark to $\Lambda$ fragmentation functions can be written as

$$
\begin{gathered}
\Delta D_{u_{v}}^{\Lambda}(z)=\Delta D_{d_{v}}^{\Lambda}(z)=\frac{1}{12} \tilde{a}_{S}^{(u)}(z)-\frac{1}{12} \tilde{a}_{V}^{(u)}(z), \\
\Delta D_{s_{v}}^{\Lambda}(z)=\frac{1}{3} \tilde{a}_{S}^{(s)}(z),
\end{gathered}
$$

and

$$
\begin{gathered}
\delta D_{u_{v}}^{\Lambda}(z)=\delta D_{d_{v}}^{\Lambda}(z)=\frac{1}{12} \hat{a}_{S}^{(u)}(z)-\frac{1}{12} \hat{a}_{V}^{(u)}(z), \\
\delta D_{s_{v}}^{\Lambda}(z)=\frac{1}{3} \hat{a}_{S}^{(s)}(z),
\end{gathered}
$$

respectively, with

$$
\tilde{a}_{D}^{(q)}(z)=\frac{N^{2} z^{2}(1-z)^{3}}{64 \pi^{2}} \frac{\left[2\left(M_{\Lambda}+m_{q} z\right)^{2}-R^{2}(z)\right]}{R^{6}(z)}
$$

and

$$
\hat{a}_{D}^{(q)}(z)=\frac{N^{2} z^{2}(1-z)^{3}}{32 \pi^{2}} \frac{\left(M_{\Lambda}+m_{q} z\right)^{2}}{R^{6}(z)}
$$

for $D=S$ or $V$. What we are interested is not the magnitude values of the fragmentation functions but the flavor and spin structure of them which are given by the diquark model. In order to extract information on the flavor and spin structure, we introduce the flavor structure ratios

$$
\begin{aligned}
& F_{S}^{(u / s)}(z)=\frac{a_{S}^{(u)}(z)}{a_{S}^{(s)}(z)}, \\
& F_{M}^{(u / s)}(z)=\frac{a_{V}^{(u)}(z)}{a_{S}^{(s)}(z)},
\end{aligned}
$$


and the spin structure ratios

$$
\begin{aligned}
& \tilde{W}_{D}^{(q)}(z)=\frac{\tilde{a}_{D}^{(q)}(z)}{a_{D}^{(q)}(z)}, \\
& \hat{W}_{D}^{(q)}(z)=\frac{\hat{a}_{D}^{(q)}(z)}{a_{D}^{(q)}(z)},
\end{aligned}
$$

with $D=S$ or $V$. Then we can use the unpolarized fragmentation function $D_{s_{v}}^{\Lambda}(z)$ to express all other unpolarized and polarized fragmentation functions as follows

$$
\begin{gathered}
D_{u_{v}}^{\Lambda}(z)=\left[\frac{3}{4} F_{M}^{(u / s)}(z)+\frac{1}{4} F_{S}^{(u / s)}(z)\right] D_{s_{v}}^{\Lambda}(z) \\
\Delta D_{u_{v}}^{\Lambda}(z)=\frac{1}{4}\left[\tilde{W}_{S}^{(u)}(z) F_{S}^{(u / s)}(z)-\tilde{W}_{V}^{(u)}(z) F_{M}^{(u / s)}(z)\right] D_{s_{v}}^{\Lambda}(z) \\
\Delta D_{s_{v}}^{\Lambda}(z)=\tilde{W}_{S}^{(s)}(z) D_{s_{v}}^{\Lambda}(z) \\
\delta D_{u_{v}}^{\Lambda}(z)=\frac{1}{4}\left[\hat{W}_{S}^{(u)}(z) F_{S}^{(u / s)}(z)-\hat{W}_{V}^{(u)}(z) F_{M}^{(u / s)}(z)\right] D_{s_{v}}^{\Lambda}(z)
\end{gathered}
$$

and

$$
\delta D_{s_{v}}^{\Lambda}(z)=\hat{W}_{S}^{(s)}(z) D_{s_{v}}^{\Lambda}(z)
$$

In this way, the polarized quark to $\Lambda$ fragmentation functions are related to the unpolarized fragmentation functions by means of some input ratios from the diquark model calculation.

The quark-diquark description of the fragmentation function should be reasonable in large $z$ region where the valence quark contributions dominate. In small $z$ region, the sea contribution is difficult to be included in the framework of the diquark model itself. In order to optimize the shape of fragmentation functions, we adopt simple functional forms

$$
D_{s_{v}}^{\Lambda}(z)=N_{s} z^{\alpha_{s}}(1-z)^{\beta_{s}}
$$


and

$$
D_{q_{s}}^{\Lambda}(z)=D_{\bar{q}}^{\Lambda}(z)=\bar{N} z^{\bar{\alpha}}(1-z)^{\bar{\beta}}
$$

to parametrize fragmentation functions of the valence quark $D_{s_{v}}^{\Lambda}$, sea quark $D_{q_{s}}^{\Lambda}(z)$ and antiquark $D_{\bar{q}}^{\Lambda}(z)$ for $q=u, d, s$. We assume that $D_{g}^{\Lambda}, \Delta D_{g}^{\Lambda}, \Delta D_{q_{s}}^{\Lambda}, \Delta D_{\bar{q}}^{\Lambda}, \delta D_{q_{s}}^{\Lambda}$, and $\delta D_{\bar{q}}^{\Lambda}$ at the initial scale are zero and they may appear due to the QCD evolution. Hence, the input unpolarized and polarized $q \rightarrow \Lambda$ fragmentation functions can be written as

$$
\begin{gathered}
D_{q}^{\Lambda}(z)=D_{q_{v}}^{\Lambda}(z)+D_{q_{s}}^{\Lambda}(z), \\
\Delta D_{q}^{\Lambda}(z)=\Delta D_{q_{v}}^{\Lambda}(z),
\end{gathered}
$$

and

$$
\delta D_{q}^{\Lambda}(z)=\delta D_{q_{v}}^{\Lambda}(z)
$$

For a fit to the experimental data, the fragmentation functions have to be evolved to the scale of the experiments. We use the evolution package of Ref. [34] suitable modified for the evolution of fragmentation functions in leading order, taking the input scale $Q_{0}^{2}=M_{\Lambda}^{2}$ and the QCD scale parameter $\Lambda_{Q C D}=0.3 \mathrm{GeV}$. Furthermore, in order to express the inclusive cross section and polarization for the $\Lambda$ production in $e^{+} e^{-}$annihilation, we introduce the following quantities

$$
\begin{gathered}
\hat{A}_{q}=2 \chi_{2}\left(v_{e}^{2}+a_{e}^{2}\right) v_{q} a_{q}-2 e_{q} \chi_{1} a_{q} v_{e}, \\
\hat{C}_{q}=e_{q}^{2}-2 \chi_{1} v_{e} v_{q} e_{q}+\chi_{2}\left(a_{e}^{2}+v_{e}^{2}\right)\left(a_{q}^{2}+v_{q}^{2}\right),
\end{gathered}
$$

with

$$
\begin{aligned}
\chi_{1} & =\frac{1}{16 \sin ^{2} \theta_{W} \cos ^{2} \theta_{W}} \frac{s\left(s-M_{Z}^{2}\right)}{\left(s-M_{Z}^{2}\right)^{2}+M_{Z}^{2} \Gamma_{Z}^{2}}, \\
\chi_{2} & =\frac{1}{256 \sin ^{4} \theta_{W} \cos ^{4} \theta_{W}} \frac{s^{2}}{\left(s-M_{Z}^{2}\right)^{2}+M_{Z}^{2} \Gamma_{Z}^{2}},
\end{aligned}
$$




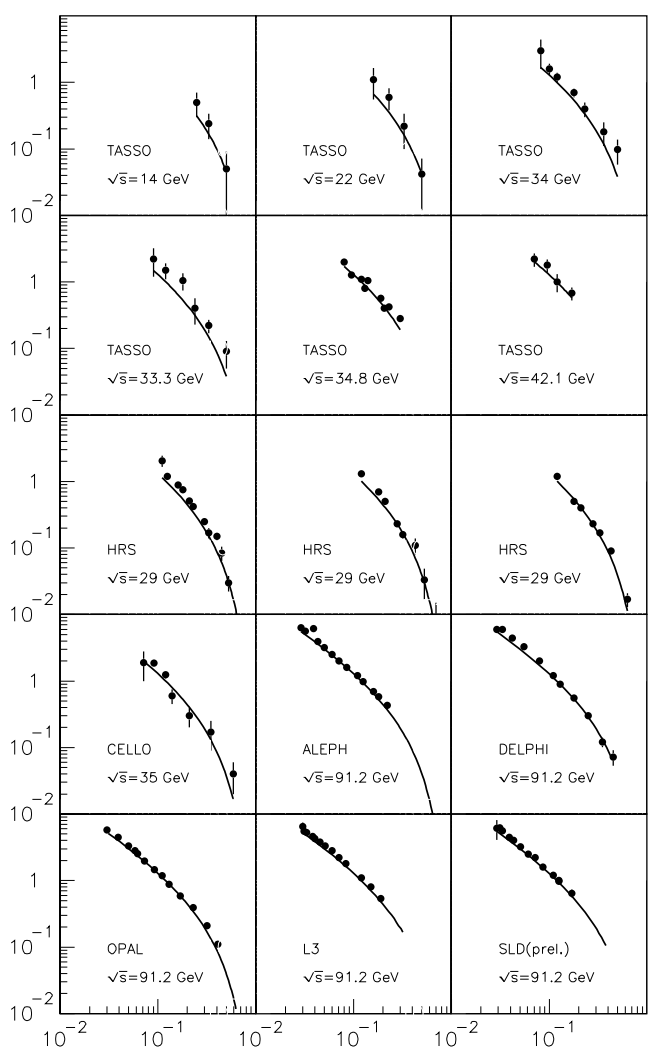

Figure 1: The comparison of our results for the $x_{E}$ dependence of the inclusive $\Lambda$ production cross section $\left(1 / \sigma_{t o t}\right) d \sigma / d x_{E}$ in $e^{+} e^{-}$annihilation and the experimental data [35-40].

$$
\begin{gathered}
a_{e}=-1 \\
v_{e}=-1+4 \sin ^{2} \theta_{W} \\
a_{q}=2 T_{3 q}, \\
v_{q}=2 T_{3 q}-4 e_{q} \sin ^{2} \theta_{W},
\end{gathered}
$$

where $T_{3 q}=1 / 2$ for $u$, while $T_{3 q}=-1 / 2$ for $d, s$ quarks, $N_{c}=3$ is the color number, $e_{q}$ is the charge of the quark in units of the proton charge, $s$ is the total centerof-mass (c.m.) energy squared, $\theta$ is the angle between the outgoing quark and the incoming electron, $\theta_{W}$ is the Weinberg angle, and $M_{Z}$ and $\Gamma_{Z}$ are the mass and width of $Z^{0}$. In the quark-parton model, the differential cross section for the semi-inclusive 

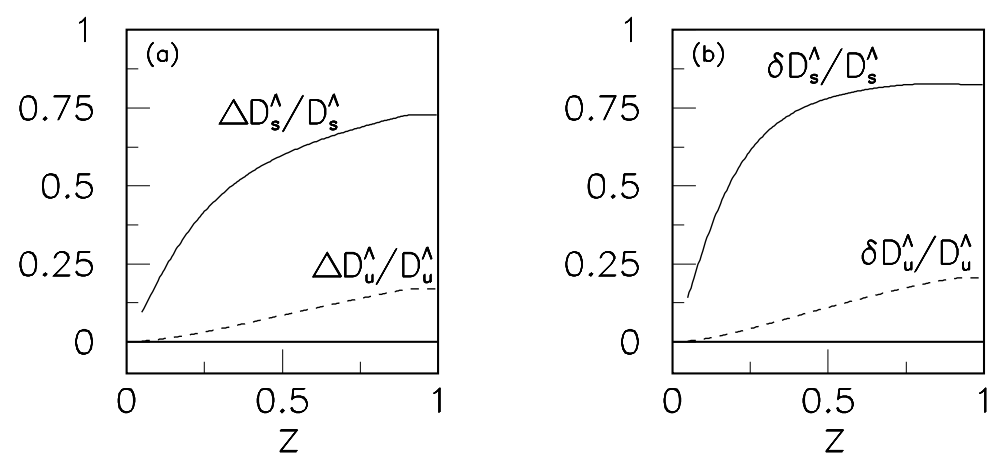

Figure 2: The spin structure of the $\Lambda$ fragmentation functions at $Q^{2}=4 \mathrm{GeV}^{2}$ : (a) $\Delta D_{q}^{\Lambda} / D_{q}^{\Lambda}$ and (b) $\delta D_{q}^{\Lambda} / D_{q}^{\Lambda}$.

$\Lambda$ production process $e^{+} e^{-} \rightarrow \Lambda+X$ can be expressed to leading order

$$
\frac{1}{\sigma_{t o t}} \frac{d \sigma}{d x_{E}}=\frac{\sum_{q} \hat{C}_{q}\left[D_{q}^{\Lambda}\left(x_{E}, Q^{2}\right)+D_{\bar{q}}^{\Lambda}\left(x_{E}, Q^{2}\right)\right]}{\sum_{q} \hat{C}_{q}}
$$

where $x_{E}=2 E_{\Lambda} / \sqrt{s}$ with $E_{\Lambda}$ being the energy of the produced $\Lambda$ in the $e^{+} e^{-}$c.m. frame, and $\sigma_{t o t}$ is the total cross section for the process.

We perform the leading order fit since the analysis in Ref. 15 shows that the leading order fit can arrive at the same fitting quality as the next-to-leading order fit. By fitting the inclusive unpolarized $\Lambda$ production data in $e^{+} e^{-}$annihilation, the optimal parameters in Eqs. (21)-(22) are obtained as $N_{s}=2.5, \alpha_{s}=0.7, \beta_{s}=3.0$,

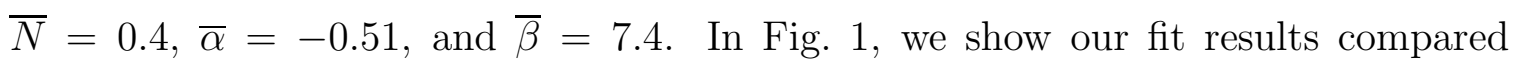
with the experimental data [35-40]. In addition, we show the spin structure of the $\Lambda$ fragmentation functions in Fig. 2. From Fig. 2, one can see that the transverse and helicity structure of the $\Lambda$ fragmentation functions in the diquark model are very similar each other.

\section{$3 \quad$ Longitudinal $q \rightarrow \Lambda$ polarization transfers}

In order to check the obtained $\Lambda$ fragmentation functions, we apply them to predict the spin observables in various $\Lambda$ production processes. 


\section{1 $\Lambda$ polarization in $e^{+} e^{-}$annihilation}

Due to the interference between the vector and axial vector coupling in the standard model for electroweak interaction, quarks produced in $e^{+} e^{-}$-annihilation at the Z-pole are polarized. The polarization of the initial quarks before the hadronization can be given according to the standard electroweak theory. With the $q \rightarrow \Lambda$ fragmentation functions, the $\Lambda$-polarization in $e^{+} e^{-}$-annihilation can be expressed as

$$
P^{\Lambda}=-\frac{\sum_{q} \hat{A}_{q}\left[\Delta D_{q}^{\Lambda}(z)-\Delta D_{\bar{q}}^{\Lambda}(z)\right]}{\sum_{q} \hat{C}_{q}\left[D_{q}^{\Lambda}(z)+D_{\bar{q}}^{\Lambda}(z)\right]} .
$$

where $\hat{A}_{q}$ and $\hat{C}_{q}(q=u, d$ and $s)$ are given in (26) and (27), respectively. At the Z-pole, we have the values for $\hat{A}_{u} \simeq 128, \hat{A}_{d}=\hat{A}_{s} \simeq 231, \hat{C}_{u} \simeq 192$, and $\hat{C}_{d}=\hat{C}_{s} \simeq 247$. In addition, one can see from Fig. 2 that $\Delta D_{s}^{\Lambda}(z) / D_{s}^{\Lambda}(z)$ is much larger than $\Delta D_{u}^{\Lambda}(z) / D_{u}^{\Lambda}(z)=\Delta D_{d}^{\Lambda}(z) / D_{d}^{\Lambda}(z)$. Therefore, the polarization of the produced $\Lambda$ at the Z-pole is mainly controlled by the polarized $s \rightarrow \Lambda$ fragmentation, i.e., $C_{s}(z)=\Delta D_{s}^{\Lambda}(z) / D_{s}^{\Lambda}(z)$. In the present analysis, $C_{s}(z)$ is proposed based on the diquark model with a clear physics motivation. Therefore, one can contrast the qualitative features of the spin observable in this process with the fragmentation functions as shown in Fig. 2. Our theoretical prediction for the $\Lambda$ polarization at the $Z$-pole is shown in Fig. 3(a) together with the experimental data. From Fig. 3(a), we can see that the calculated $\Lambda$ polarization is consistent with the experimental data, which indicates that the helicity structure of the $\Lambda$ fragmentation functions proposed in the diquark model with a clear physics motivation seems to be more realistic than that with some limit assumptions [15].

\subsection{Spin transfer to $\Lambda$ in polarized charged lepton DIS}

In the charged lepton deeply inelastic scattering process, if the initial charged lepton beam is polarized, the outgoing quark should also be polarized. The polarization of the outgoing quark depends on $P_{B}$, the polarization of the initial lepton beam. There should be a factor $D(y)$ which relates the polarization of the outgoing quark with 

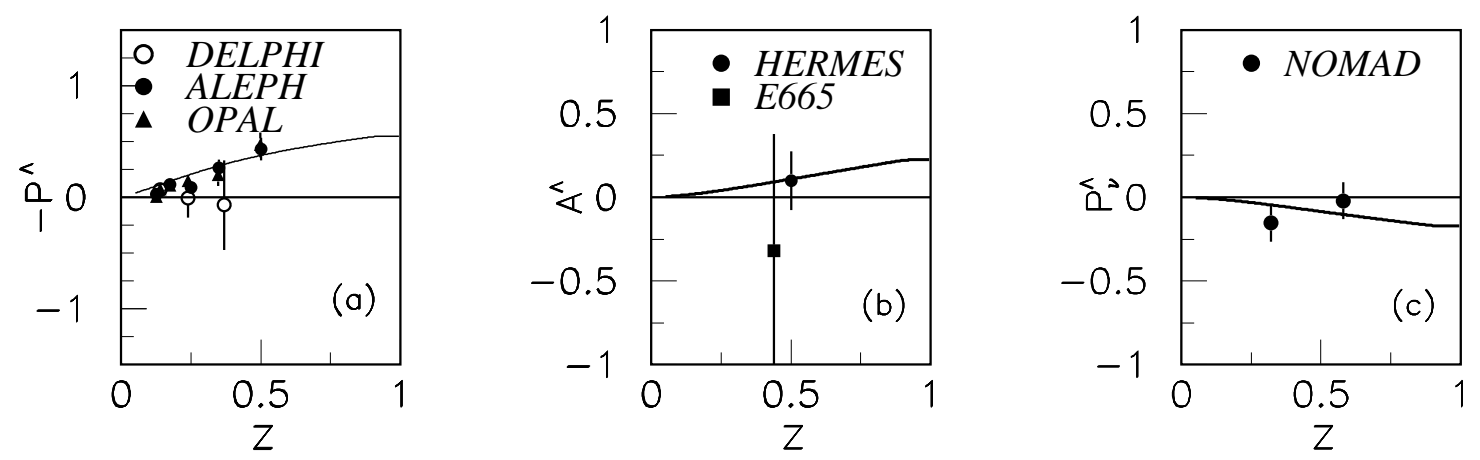

Figure 3: (a) The comparison of the experimental data [1-3] for the longitudinal $\Lambda$ polarization $P^{\Lambda}$ in $e^{+} e^{-}$-annihilation at the $Z$-pole with our theoretical prediction; (b) The $z$-dependence of the $\Lambda$ spin transfer in electron or positron (muon) DIS; (c) The $z$-dependence of the $\Lambda$ polarization in the neutrino DIS process.

that of the initial lepton. The factor $D(y)$ with its explicit expression

$$
D(y)=\frac{1-(1-y)^{2}}{1+(1-y)^{2}}
$$

is commonly referred to as the longitudinal depolarization factor of the virtual photon with respect to the parent lepton. Here $y=\nu / E$ is the fraction of the incident lepton's energy that is transferred to the hadronic system by the virtual photon. Therefore, for a longitudinally polarized charged lepton beam and an unpolarized target, the $\Lambda$ polarization along its own momentum axis is given in the quark parton model by [16]

$$
P_{\Lambda}(x, y, z)=P_{B} D(y) A^{\Lambda}(x, z)
$$

where

$$
A^{\Lambda}(x, z)=\frac{\sum_{q} e_{q}^{2}\left[q^{N}\left(x, Q^{2}\right) \Delta D_{q}^{\Lambda}\left(z, Q^{2}\right)+(q \rightarrow \bar{q})\right]}{\sum_{q} e_{q}^{2}\left[q^{N}\left(x, Q^{2}\right) D_{q}^{\Lambda}\left(z, Q^{2}\right)+(q \rightarrow \bar{q})\right]}
$$

is the longitudinal spin transfer to the $\Lambda$. In Eq. (38), $q^{N}\left(x, Q^{2}\right)$, the quark distribution of the proton, is adopted as the CTEQ5 set 1 parametrization form 41] in our numerical calculations. Our prediction result is shown in Fig. 3(b). Note that for HERMES data [4] the $\Lambda$ polarization is measured along the virtual-photon momentum, whereas for E665 data [5] it is measured along the virtual-photon spin. 
The averaged value of the Bjorken variable is chosen as $x=0.1$ (corresponding to the HERMES averaged value) and the calculated result is not sensitive to a different choice of $x$ in the small $x$ region (for example, $x=0.005$ corresponding to the E665 averaged value). $Q^{2}=4 \mathrm{GeV}^{2}$ is used and the $Q^{2}$ dependence of the result is very weak. The prediction is compatible with the available experimental data in the medium $z$ region, which suggests that the $u$ and $d$ quark to the $\Lambda$ fragmentation functions are likely positive polarized in the medium and large $z$ region. This prediction is consistent with our previous results based on the light cone $\mathrm{SU}(6)$ quark diquark spectator model and the pQCD counting rules analysis [13], and the statistical model [14]. However, the present prediction shows the weaker $z$ dependence in the large $z$ region than the results in Ref. [13]. We will show in the next subsection that the NOMAD data [6] on the $\Lambda$ polarization in neutrino DIS seems to favor the weaker $z$ dependence prediction.

Actually, the Lambdas seen at HERMES arise not only from the fragmentation of $u, d$, or $s$ quarks, but also from electroproduction of heavier hyperons which subsequently decay to Lambdas. The main contributions from decays are the $\Sigma^{0} \rightarrow \Lambda \gamma$ and $\Sigma^{*} \rightarrow \Lambda \pi$. The $\Sigma^{0}$ decays electromagnetically. Its decay can not be included in any strong interaction fragmentation function and the $\Lambda$ 's produced via its production and decay must be considered separately. It has been shown that the produced $\Lambda$ which comes from the decay of a heavier hyperon is also polarized if the heavier hyperon was polarized before its decay. In our present analysis, we only included the directly produced $\Lambda$. The contributions from the heavier hyperon decay are not easily included theoretically at the moment although they would change the interpretation of the observed polarization in this process. However, we have noticed that the electroproduction of hyperons is dominated from the $u$ quark due to the charge factor for the $u$ quark and that $u^{\uparrow}(d s)_{0,0} \rightarrow \Lambda, u^{\uparrow}(d s)_{0,0} \rightarrow \Sigma^{0}$ and $u^{\uparrow}(d s)_{1,0} \rightarrow \Sigma^{*}$ predicted by the diquark model have the similar $z$ dependence. So it is expected that the spin transfer in charged lepton DIS with the effect of the $\Sigma^{0}$ and $\Sigma^{*}$ decays can be equivalently expressed by the direct hadronized $\Lambda$ spin transfer multiplied by a factor. Hence, the qualitative feature of the predicted spin transfer is also expected to be retained after the heavier hyperon decay contributions are further included. 


\section{3 $\Lambda$ polarization in neutrino DIS}

One advantage of the neutrino (anti-neutrino) DIS process is that the scattering of a neutrino beam on a hadronic target provides a source of polarized quarks with specific flavor structure. According to the weak interaction theory, the charged current weak interaction selects only left-handed quarks (right-handed antiquarks), so the polarization of the fragmenting quarks is $P_{q}=-1$. The above particular property makes the neutrino process an ideal laboratory to study the flavor-dependence of quark to hadron fragmentation functions, especially in the polarized case. We find that the $\Lambda$ polarization in the neutrino DIS process can also be used to extract information on the $u \rightarrow \Lambda$ polarization transfer.

The longitudinal polarizations of the $\Lambda$ in its momentum direction, for the $\Lambda$ in the current fragmentation region, can be expressed as 223

$$
P_{\nu}^{\Lambda}(x, y, z)=-\frac{\left[d^{N}(x)+\varpi s^{N}(x)\right] \Delta D_{u}^{\Lambda}(z)-(1-y)^{2} \bar{u}^{N}(x)\left[\Delta D \frac{\Lambda}{d}(z)+\varpi \Delta D \frac{\Lambda}{s}(z)\right]}{\left[d^{N}(x)+\varpi s^{N}(x)\right] D_{u}^{\Lambda}(z)+(1-y)^{2} \bar{u}^{N}(x)\left[D \frac{\Lambda}{d}(z)+\varpi D \frac{\Lambda}{s}(z)\right]}
$$

where the terms with the factor $\varpi=\sin ^{2} \theta_{c} / \cos ^{2} \theta_{c}\left(\theta_{c}\right.$ is the Cabibbo angle) represent Cabibbo suppressed contributions. The NOMAD data [6] on the $\Lambda$ polarization in the neutrino DIS process, which has much better precision than the data on the longitudinal spin transfer to the $\Lambda$ in polarized charged lepton DIS, puts further constraints on the allowed form of the fragmentation functions, primarily for $u \rightarrow \Lambda$ transitions.

In Fig. B(c), we present our prediction of the $z$-dependence of the $\Lambda$ polarization in the neutrino DIS process. In the obtained result, we adopted the CTEQ5 set 1 quark distributions [41] for the target proton at $Q^{2}=4 \mathrm{GeV}^{2}$ with the Bjorken variable $x$ integrated over $0.02 \rightarrow 0.4$ and $y$ integrated over $0 \rightarrow 1$. The very recent NOMAD data [6] seems to support our prediction that the $z$-dependence of $\Lambda$ polarization in neutrino DIS is not strong. 


\section{Nucleon transversity distributions}

Within the framework of the quark diquark model [10], if any one of the quarks is probed, re-organize the other two quarks in terms of two quark wave functions with spin 0 or 1 (scalar and vector diquarks), i.e., the diquark serves as an effective particle which is called the spectator. Some non-perturbative effects such as gluon exchanges in the hadronic debris can be effectively taken into account by the mass of the diquark spectator. More explicitly, the unpolarized valence quark distributions in the proton can be expressed as

$$
\begin{gathered}
u_{v}^{N}(x)=\frac{1}{2} A_{S}^{(u)}(x)+\frac{1}{6} A_{V}^{(u)}(x), \\
d_{v}^{N}(x)=\frac{1}{3} A_{V}^{(d)}(x),
\end{gathered}
$$

where $A_{D}^{(q)}(x)(D=S$ or $V)$, which is the probability of finding a quark $q$ to be scattered while the spectator is in the diquark state $D$, can be expressed in the quark-diquark model as [10]

$$
A_{D}^{(q)}(x)=\frac{N^{2}(1-x)^{3}}{32 \pi^{2}} \frac{\left[2\left(x M_{p}+m_{q}\right)^{2}+\hat{R}^{2}(x)\right]}{\hat{R}^{6}(x)}
$$

with

$$
\hat{R}(x)=\sqrt{\Lambda_{0}^{2}(1-x)+x m_{D}^{2}-x(1-x) M_{p}^{2}},
$$

where $M_{p}$ and $m_{D}(D=S$ or $V)$ are the mass of the proton and a diquark, respectively. Similarly, the polarized quark distributions of the proton can be written as

$$
\begin{gathered}
\Delta u_{v}^{N}(x)=\frac{1}{2} \tilde{A}_{S}^{(u)}(x)-\frac{1}{18} \tilde{A}_{V}^{(u)}(x), \\
\Delta d_{v}^{N}(x)=-\frac{1}{9} \tilde{A}_{V}^{(d)}(x)
\end{gathered}
$$

for the helicity distributions, and 


$$
\begin{gathered}
\delta u_{v}^{N}(x)=\frac{1}{2} \hat{A}_{S}^{(u)}(x)-\frac{1}{18} \hat{A}_{V}^{(u)}(x), \\
\delta d_{v}^{N}(x)=-\frac{1}{9} \hat{A}_{V}^{(d)}(x),
\end{gathered}
$$

for the transverse distributions with

$$
\tilde{A}_{D}^{(q)}(x)=\frac{N^{2}(1-x)^{3}}{32 \pi^{2}} \frac{\left[2\left(x M_{p}+m_{q}\right)^{2}-\hat{R}^{2}(x)\right]}{\hat{R}^{6}(x)}
$$

and

$$
\hat{A}_{D}^{(q)}(x)=\frac{N^{2}(1-x)^{3}}{16 \pi^{2}} \frac{\left(x M_{p}+m_{q}\right)^{2}}{\hat{R}^{6}(x)}
$$

for $D=S$ or $V$. A detailed analysis of the helicity distributions has been done in Ref. [10]. Here, we focus our attention on the quark transverse distributions of the nucleon.
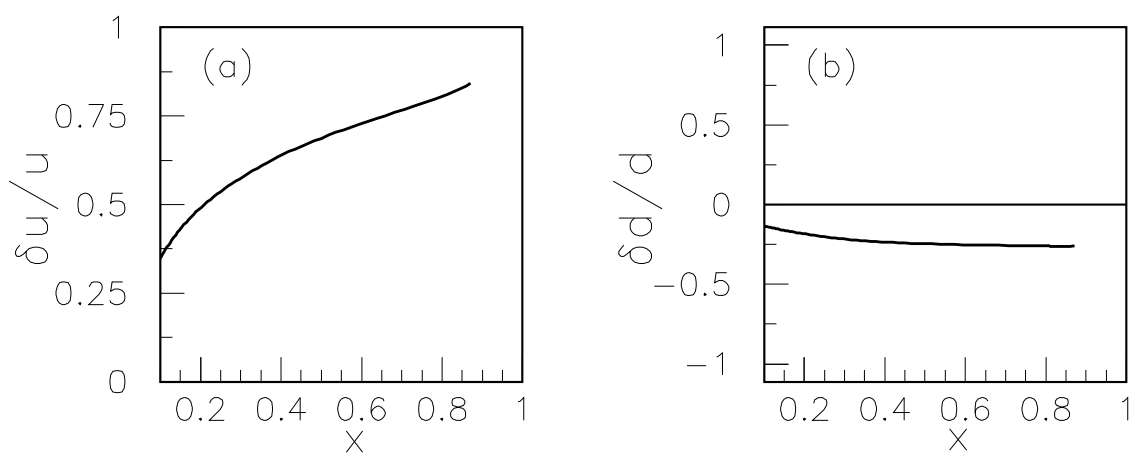

Figure 4: The quark spin structure of the transversely polarized proton at $Q^{2}=$ $4 \mathrm{GeV}^{2}$ : (a) $\delta u / u$ and (b) $\delta d / d$.

The quark-diquark model with a simple form factor for the nucleon-quark-diquark vertex can provide good relations between different quantities in which the uncertainties in the model can be canceled between each other. It is impractical to expect a good description of the absolute magnitude and shape for a physical quantity. However, following Ref. [42], we may use some useful relations to connect the unmeasured quantities with the measured quantities. For example, we may use the following 
relation to connect the quark transverse distributions with the quark unpolarized distributions

$$
\begin{gathered}
\delta u_{v}^{N}(x)=\left[u_{v}^{N}(x)-\frac{1}{2} d_{v}^{N}(x)\right] \hat{W}_{S}^{(u)}(x)-\frac{1}{6} d_{v}^{N}(x) \hat{W}_{V}^{(u)}(x) \\
\delta d_{v}^{N}(x)=-\frac{1}{3} d_{v}^{N}(x) \hat{W}_{V}^{(d)}(x),
\end{gathered}
$$

with

$$
\hat{W}_{D}^{(q)}(x)=\frac{\hat{A}_{D}^{(q)}(x)}{A_{D}^{(q)}(x)} .
$$

We can use the unpolarized valence quark distributions $u_{v}^{N}(x)$ and $d_{v}^{N}(x)$ at an initial scale from one set of quark distribution parametrizations as inputs to calculate the quark transverse distributions with $\hat{W}_{D}^{(q)}(x)$ from the diquark model calculation. In this way we can make more reliable prediction for the absolute magnitude and shape of a physical quantity than directly from the model calculation. It is worth to emphasize that Eq. (50) can only serve as the connection between the transverse polarized quark distributions and the unpolarized quark distributions at the initial scale $\left(\sim 1 \mathrm{GeV}^{2}\right)$. The QCD evolution for the transverse quark distributions is quite different from that for the unpolarized quark distributions since the transverse distributions receive no contribution from transversely polarized gluons. In the following numerical calculations, the CTEQ5 set 1 parametrization forms [41] are adopted as inputs for the unpolarized quark distributions of the nucleon. In Fig. 田, we show the transversely polarized structure for the $u$ and $d$ quarks in a transversely polarized proton at $Q^{2}=4 \mathrm{GeV}^{2}$.

\section{Azimuthal spin asymmetry in pion electropro- duction}

Semi-inclusive pion production in deep inelastic scattering of leptons off a polarized nucleon target is a powerful tool for providing information on the spin structure of the nucleon and on parton fragmentation. The HERMES Collaboration [31] has recently reported the measurement results of single-spin asymmetries in the azimuthal 

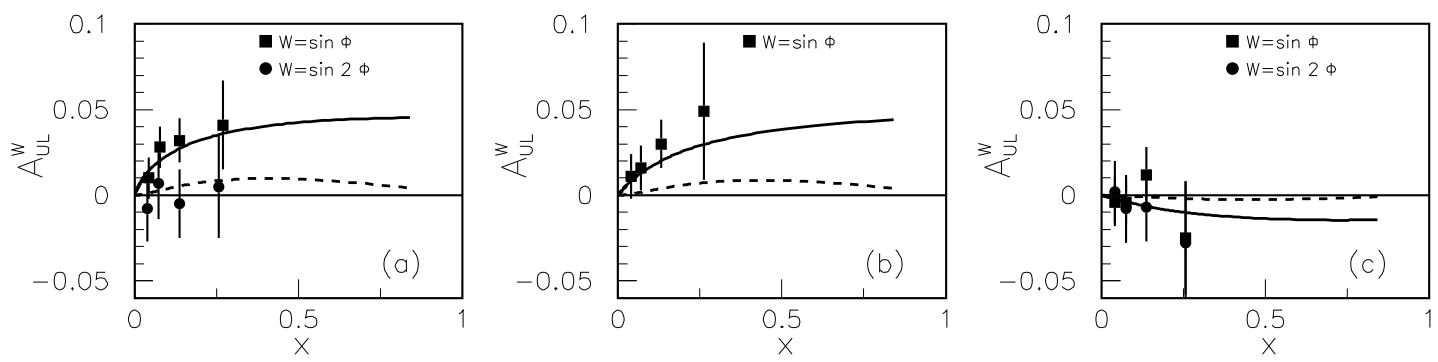

Figure 5: The analyzing powers $A_{U L}^{\sin \phi}$ and $A_{U L}^{\sin 2 \phi}$ for (a) $\pi^{+}$, (b) $\pi^{0}$ and (c) $\pi^{-}$of semi-inclusive pion production in deep inelastic scattering of unpolarized positrons on the longitudinally polarized proton target. The solid and dashed curves correspond to $A_{U L}^{\sin \phi}$ and $A_{U L}^{\sin 2 \phi}$, respectively. The input quark distributions are from CTEQ5 set 1 parametrization forms at at $Q^{2}=4 \mathrm{GeV}^{2}$.

distribution of charged and neutral pions relative to the lepton scattering plane in deep-inelastic scattering of positrons off longitudinally polarized protons. It was found that the dependence of the single-spin azimuthal asymmetry for $\pi^{0}$ production on the Bjorken scaling variable $x$ is similar to the result for $\pi^{+}$production, while the $\pi^{-}$ asymmetry is consistent with zero within present experimental uncertainties.

The analyzing power measured by HERMES for unpolarized (U) beam and longitudinally $(\mathrm{L})$ polarized target is defined as

$$
A_{U L}^{W}=\frac{\int[\mathrm{d} \phi] W(\phi)\left\{N^{+}(\phi)-N^{-}(\phi)\right\}}{\frac{1}{2} \int[\mathrm{d} \phi]\left\{N^{+}(\phi)+N^{-}(\phi)\right\}},
$$

where $W(\phi)=\sin \phi$ or $\sin 2 \phi$ is the weighting function for picking up the Collins effect, and $N^{+}(\phi)\left(N^{-}(\phi)\right)$ is the number of events for pion production as a function of $\phi$ when the target is positively (negatively) polarized. The analyzing powers for $\pi^{+}, \pi^{0}$ and $\pi^{-}$have been measured [31], and from the data there is clear evidence for the non-zero values of $A_{U L}^{\sin \phi}$ for $\pi^{+}$and $\pi^{0}$, which indicates the azimuthal asymmetry.

The analyzing power $A_{U L}^{W}$ is a function of the pion fractional energy $z$, the Bjorken scaling variable $x$, and the pion transverse momentum $P_{\perp}$. However, what we are interested in the present investigation is the $x$ dependence of $A_{U L}^{W}$ since it can bring us information on the quark transverse distributions of the nucleon. Based on a theoretical analysis presented in Refs. [28, 43, it was found 42] that the analyzing powers $A_{U L}^{\sin \phi}$ and $A_{U L}^{\sin 2 \phi}$ are proportional to the ratios 


$$
\frac{\sum_{q} e_{q}^{2} \delta q^{N}(x)\left\langle\delta D_{q}^{\pi}(z) / z\right\rangle}{\sum_{q} e_{q}^{2} q^{N}(x)\left\langle D_{q}^{\pi}(z)\right\rangle},
$$

and

$$
\frac{x^{2} \sum_{q} e_{q}^{2}\left(\int_{x}^{1} \mathrm{~d} \xi \delta q^{N}(\xi) / \xi^{2}\right)\left\langle\delta D_{q}^{\pi}(z)\right\rangle}{\sum_{q} e_{q}^{2} q^{N}(x)\left\langle D_{q}^{\pi}(z)\right\rangle},
$$

respectively. Here $e_{q}$ is the charge of the quark with flavor $q, q^{N}(x)$ and $\delta q^{N}(x)$ are the quark unpolarized and transverse distributions of the nucleon target, and $D_{q}^{\pi}(z)$ and $\delta D_{q}^{\pi}(z)$ are the fragmentation functions to the pion $\pi$ from an unpolarized and transversely polarized quark with flavor $q$. A further detailed theoretical analysis can be found in Ref. [44]. Therefore with the inputs of $D_{q}^{\pi}(z)$ and $\delta D_{q}^{\pi}(z)$, we are able to get the quark transverse distributions $\delta q^{N}(x)$ from the measured analyzing powers. We will, following Ref. [42], consider only the contributions from the favored fragmentation functions $D_{q}^{\pi}$ and $\delta D_{q}^{\pi}$, i.e., $D(z)=D_{u}^{\pi^{+}}(z)=D_{\frac{\pi^{+}}{d}}(z)=2 D_{u}^{\pi^{0}}(z)=$ $2 D_{d}^{\pi^{0}}(z)=2 D_{\frac{\pi^{0}}{u}}(z)=2 D_{\frac{\pi^{0}}{d}}(z)=D_{d}^{\pi^{-}}(z)=D_{\frac{\pi^{-}}{u}}(z)$ and similarly for $\delta D_{q}^{\pi}$. The average values for $\left\langle D_{q}^{\pi}(z)\right\rangle,\left\langle\delta D_{q}^{\pi}(z)\right\rangle,\left\langle\delta D_{q}^{\pi}(z) / z\right\rangle$, and the corresponding parameters are also chosen the same as Ref. [42]. Therefore, with the obtained quark transverse distributions of the nucleon, we can calculate Eqs. (53) and (54). The calculated results as shown in Fig. 5 are consistent with the HERMES experimental data [31]. By contrasting the qualitative features of the nucleon transversity distributions as

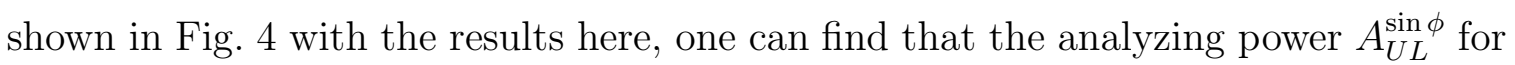
$\pi^{+}$production is mainly controlled by $\delta u / u$. It is due to the charge factor for the $u$ quark and the fact that $\pi^{+}$production is dominated by the $u \rightarrow \pi^{+}$fragmentation process. In addition, the effect of the unfavored quark to pion fragmentation functions on $A_{U L}^{\sin \phi}$ for $\pi^{+}$production is expected to be small. However, the situation of $A_{U L}^{\sin \phi}$ for $\pi^{-}$production is a little complex since the contribution of unfavored quark to pion fragmentation functions might have significant effects on $\pi^{-}$production. As for a detailed discussion, we would like to recommend our recent work in Ref. [32]. It is due to the fact that $\pi^{+}$and $\pi^{-}$productions are dominated by the $u \rightarrow \pi^{+}$ and $d \rightarrow \pi^{-}$fragmentation processes, respectively. Therefore, we can extract some useful information on the nucleon transversity distributions by means of the analyzing 

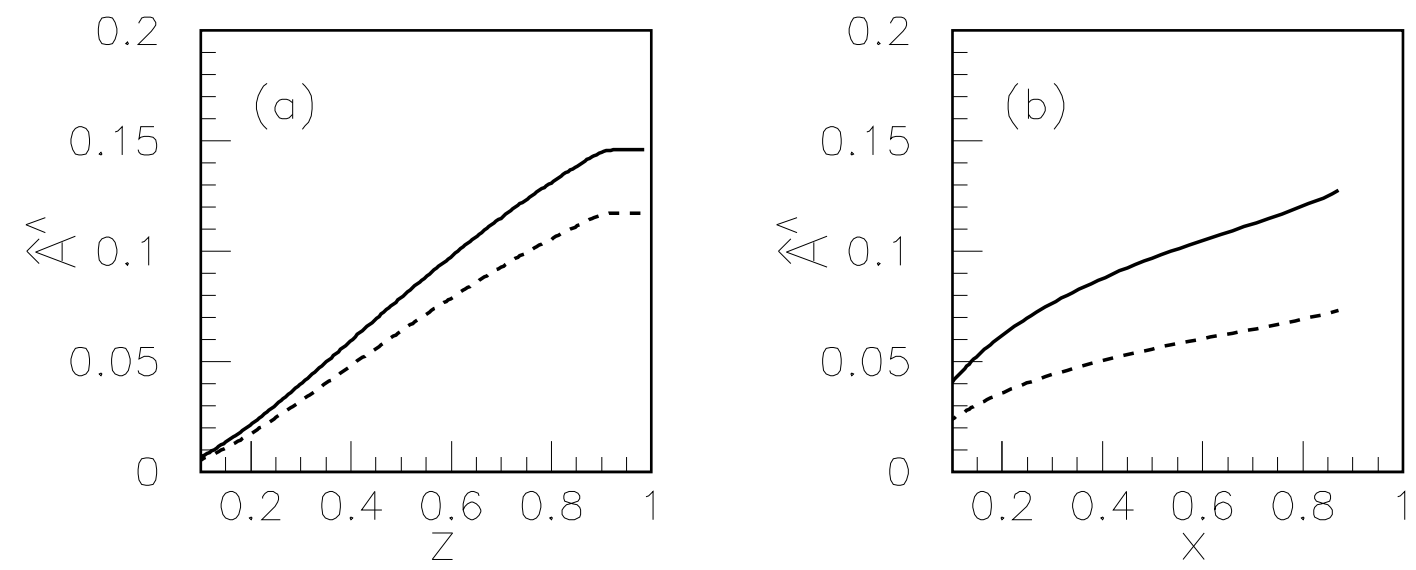

Figure 6: The $x$-integrated and $z$-integrated spin transfers $\hat{A}^{\Lambda}(x, z)$ of $\Lambda$ production in charged lepton DIS process on the transversely polarized proton target, with the integrated $x$ or $z$ range of $0.6 \rightarrow 1$ for the solid curves and $0.3 \rightarrow 1$ for the dashed curves at $Q^{2}=4 \mathrm{GeV}^{2}$.

powers $A_{U L}^{\sin \phi}$ for $\pi$ productions.

\section{$6 \quad$ Transverse $q \rightarrow \Lambda$ polarization transfers}

In addition to the azimuthal spin asymmetry in pion electroproduction, the $\Lambda$ transverse polarization in the current fragmentation region of charged lepton DIS on the transversely polarized nucleon target can also provide information on the quark transverse distribution of the target. For $\Lambda$ production in the current fragmentation region along the virtual photon direction [23], the spin transfer to the transversely polarized $\Lambda$ can be written as [16, 33]

$$
\hat{A}^{\Lambda}(x, z)=\frac{\sum_{q} e_{q}^{2} \delta q^{N}\left(x, Q^{2}\right) \delta D_{q}^{\Lambda}\left(z, Q^{2}\right)}{\sum_{q} e_{q}^{2} q^{N}\left(x, Q^{2}\right) D_{q}^{\Lambda}\left(z, Q^{2}\right)}
$$

for charged lepton DIS on a transversely polarized nucleon $N$ target. A detailed discussion about the transverse spin transfer has been given in Ref. [24]. Now we can predict this transverse spin transfer with the obtained quark distributions $q^{N}(x)$, $\delta q^{N}(x)$ for the nucleon and the $q \rightarrow \Lambda$ fragmentation functions $D_{q}^{\Lambda}(z)$ and $\delta D_{q}^{\Lambda}(z)$. 
The $x$-integrated and $z$-integrated spin transfers $\hat{A}^{\Lambda}(x, z)$ are presented in Fig. 6 . The results can be easily understood by contrasting the qualitative features of the transversely polarized $q \rightarrow \Lambda$ fragmentation functions as shown in Fig. 2(b) with the $z$ dependence of $\hat{A}^{\Lambda}$ in Fig. 6(a) and the features of the nucleon transversity distributions in Fig. $6\left(\right.$ a) with the $x$ dependence of $\hat{A}^{\Lambda}$ in Fig. 6(b). We will have some more detailed discussions in the next section. After all, it turns out that the $x$-integrated and $z$ integrated $\hat{A}^{\Lambda}(x, z)$ can provide us more information on the $q \rightarrow \Lambda$ fragmentation functions and the quark transverse distributions of the nucleon, respectively.

\section{Discussion and summary}

The $\Lambda$ polarization $P^{\Lambda}$ in $e^{+} e^{-}$annihilation at the Z-pole provides us information on the polarized $s$ quark to $\Lambda$ fragmentation function since it is dominated by the $s \rightarrow \Lambda$ fragmentation. The spin transfer $A^{\Lambda}$ in $\Lambda$ electroproduction is dominated by the $u \rightarrow \Lambda$ fragmentation due to the charge factor for the $u$ quark, i.e., Eq. (38) can be approximated by

$$
A^{\Lambda} \simeq \frac{\Delta D_{u}^{\Lambda}\left(z, Q^{2}\right)}{D_{u}^{\Lambda}\left(z, Q^{2}\right)} .
$$

The $d$ quark spin transfer to the $\Lambda$ is expected to be equal to that of the $u$ quark because of the isospin symmetry. Consequently, $\Lambda$ electroproduction in the current fragmentation region is most sensitive to the ratio $\Delta D_{u}^{\Lambda} / D_{u}^{\Lambda}=\Delta D_{d}^{\Lambda} / D_{d}^{\Lambda}$. Therefore, our consistent descriptions of the experimental data on the $\Lambda$ polarization $P^{\Lambda}$ and the spin transfer $A^{\Lambda}$ indicate that the $q \rightarrow \Lambda$ fragmentation functions obtained in the diquark model are reasonable. According to Eq. (56), the available HERMES experimental data [4] on the spin transfer suggests that the $u$ and $d$ quark to the $\Lambda$ fragmentation functions are likely positive polarized in medium and large $z$ region. Furthermore, the very recent NOMAD data [6] on the $\Lambda$ polarization in the neutrino DIS process, which is also dominated by the $u \rightarrow \Lambda$ fragmentation, supports the above information. However, despite the experimental uncertainties, the E665 experiment [5] seems to show negative $\Lambda$ polarizations in low $z$ region where the sea quark 
contribution dominates. Unfortunately, the polarized sea quark to $\Lambda$ fragmentation can neither be constructed within the framework of the diquark model nor be extracted from the available experimental data. A further study on the small $z$ region behavior of the $q \rightarrow \Lambda$ polarization transfer is required.

The analyzing power $A_{U L}^{\sin \phi}$ for $\pi^{+}$production is dominated by the $u$ quark from the fragmentation of $u \rightarrow \pi^{+}$. Therefore, the analyzing power $A_{U L}^{\sin \phi}$ for $\pi^{+}$production with summed over $z$ can provide information on the $u$ quark transverse distributions of the target nucleon. The increase of $A_{U L}^{\sin \phi}$ with increasing $x$ as shown in the HERMES data [31 for $\pi^{+}$and $\pi^{0}$ productions suggests that single-spin asymmetries are with valence quark distributions. On the other hand, the spin transfer $\hat{A}^{\Lambda}(x, z)$ of $\Lambda$ production in charged lepton DIS process on the transversely polarized proton target is also dominated by the $u \rightarrow \Lambda$ spin transfer due to the charge factor for the $u$ quark. Eq. (55) can be approximately reduced as

$$
\hat{A}^{\Lambda}(x, z) \simeq \frac{\delta u^{N}\left(x, Q^{2}\right) \delta D_{u}^{\Lambda}\left(z, Q^{2}\right)}{u^{N}\left(x, Q^{2}\right) D_{u}^{\Lambda}\left(z, Q^{2}\right)} .
$$

Hence, with $\delta u^{N}(x) / u^{N}(x)$ from the power analyzing $A_{U L}^{\sin \phi}$ for $\pi^{+}$production, $\delta D_{u}^{\Lambda} / D_{u}^{\Lambda}$ can be extracted from the $x$-integrated $\hat{A}^{\Lambda}(x, z)$. We also present the spin transfers integrated over different $z$ regions in Fig. 6 . We find that the $x$ dependence of the $z$-integrated $\hat{A}^{\Lambda}(x, z)$ is mainly shaped by $\delta u^{N}(x) / u^{N}(x)$ (see Fig. 四(a) and Fig. G(b)), as well as the $x$ dependence of the analyzing power $A_{U L}^{\sin \phi}$ for the $\pi^{+}$production (see Fig. $\mathbb{A}(\mathrm{a})$ and Fig. $5(\mathrm{a}))$. The magnitude values of the $z$-integrated $\hat{A}^{\Lambda}(x, z)$ and the analyzing power $A_{U L}^{\sin \phi}$ for the $\pi^{+}$production are sensitive to the $z$-integrated value of the fragmentation functions for the produced $\Lambda$ and $\pi^{+}$, respectively. Therefore, in order to make a mutual check for the transversely polarized $q \rightarrow \Lambda$ fragmentation functions and the transverse quark distributions of the nucleon, it is very significant to perform the measurement of the transverse spin transfer $\hat{A}^{\Lambda}(x, z)$ over various ranges of $x$ and $z$, as well as further precision measurement of $A_{U L}^{\sin \phi}$ for pion production.

In summary, based on the available unpolarized $\Lambda$ production data in $e^{+} e^{-}$annihilation, we extracted a set of $q \rightarrow \Lambda$ fragmentation functions with the flavor and spin structure from the quark-diquark model. It is found that the longitudinal spin 
structure of the obtained $\Lambda$ fragmentation functions is supported by the all available experimental data on the longitudinal $\Lambda$ polarization. Within the same framework, we proposed a set of transverse quark distributions of the nucleon and obtained consistent descriptions of the available HERMES data on the azimuthal spin asymmetry in pion electroproduction. In order to make a mutual check of the quark transverse distributions of the nucleon and the transversely polarized quark to $\Lambda$ fragmentation functions, we emphasized the significance of the measurement on spin transfer to the transversely polarized $\Lambda$ in the charged lepton DIS on a transversely polarized nucleon target. The investigation on the $q \rightarrow \Lambda$ fragmentation functions and the quark transverse distributions of the nucleon is important for enriching our knowledge of hadron structure and hadronization mechanism.

Acknowledgments: I am grateful to P. J. Mulders and J. Rodrigues for their kindness correspondence about the quark-diquark model. This work is stimulated by my other cooperative work with Bo-Qiang Ma, Ivan Schmidt, and Jacques Soffer. I also would like to express my great thanks to them for their encouragements and valuable comments. In addition, this work is partially supported by National Natural Science Foundation of China under Grant Number 19875024 and by Fondecyt (Chile) project 3990048 . 


\section{References}

[1] ALEPH Collaboration, D. Buskulic et al, Phys. Lett. B 374 (1996) 319.

[2] DELPHI Collaboration, Report No.DELPHI 95-86 PHYS 521, CERN-PPE-95172, presented at the EPS-HEP 95 conference, Brussels, 1995.

[3] OPAL Collaboration, K. Ackerstaff et al, Eur. Phys. J. C 2 (1998) 49.

[4] HERMES Collaboration, A. Airapetian et al., hep-ex/9911017.

[5] E665 Collaboration, M.R. Adams et al., Eur. Phys. J. C 17 (2000) 263.

[6] NOMAD Collaboration, P. Astier et al., Nucl. Phys. B 588 (2000) 3; D.V. Naumov, hep-ph/0101325.

[7] G. Gustafson and J. Häkkinen, Phys. Lett. B 303 (1993) 350.

[8] M. Burkardt and R.L. Jaffe, Phys. Rev. Lett. 70 (1993) 2537.

[9] W. Lu and B.-Q. Ma, Phys. Lett. B 357 (1995) 419;

W. Lu, Phys. Lett. B 373 (1996) 223;

J. Ellis, D. Kharzeev, and A. Kotzinian, Z. Phys. C 69 (1996) 467.

[10] R. Jakob, P.J. Mulders, J. Rodrigues, Nucl. Phys. A 626 (1997) 937;

M. Nzar and P. Hoodbhoy, Phys. Rev. D 51 (1995) 32.

[11] J. J. Yang, Phys. Rev. D 64, (2001) 074010.

[12] C. Boros, T. Londergan, and A.W. Thomas, Phys. Rev. D 61 (2000) 014007.

[13] B.Q. Ma, I. Schmidt, and J.J. Yang, Phys. Lett. B 477 (2000) 107; Phys. Rev. D $61(2000) 034017$.

[14] J.J. Yang, Phys. Lett. B 512 (2001) 57, hep-ph/0107222.

[15] D.de Florian, M. Stratmann, and W. Vogelsang, Phys. Rev. D 57 (1998) 5811. 
[16] R.L. Jaffe, Phys. Rev. D 54 (1996) R6581.

[17] A. Kotzinian, A. Bravar, and D.von Harrach, Eur. Phys. J. C 2 (1998) 329.

[18] D.de Florian, M. Stratmann, and W. Vogelsang, Phys. Rev. Lett. 81 (1998) 530.

[19] C. Boros and Z. Liang, Phys. Rev. D 57 (1998) 4491.

[20] B.Q. Ma and J. Soffer, Phys. Rev. Lett. 82 (1999) 2250.

[21] C. Boros and A.W. Thomas, Phys. Rev. D 60, (1999) 074017; Phys. Rev. D 62 (2000) 014021.

[22] D. Ashery and H.J. Lipkin, Phys. Lett. B 469 (1999) 263.

[23] B.Q. Ma, I. Schmidt, J. Soffer, and J.J. Yang, hep-ph/0001259, Eur. Phys. J. C $16(2000) 657$.

[24] B.Q. Ma, I. Schmidt, J. Soffer, and J.J. Yang, hep-ph/0005210, Phys. Lett. B 489 (2000) 293; Phys. Rev. D 62 (2000) 114009; Phys. Rev. D 64 (2001) 014017, Erratum-ibid.D 64, 099901 (2001).

[25] C. Liu and Z. Liang, Phys. Rev. D 62 (2000) 094001.

[26] J. Collins, Nucl. Phys. B 396 (1993) 161.

[27] A.M. Kotzinian, Nucl. Phys. B 441 (1995) 234.

[28] P.J. Mulders and R.D. Tangerman, Nucl. Phys. B 461 (1996) 197.

[29] R. Jaffe, X. Jin, and J. Tang, Phys. Rev. Lett. 80 (1998) 1166.

[30] M. Anselmino, B. Boglione, and F. Murgia, Phys. Lett. B 362 (1995) 164.

[31] HERMES Collaboration, A. Airapetian et al., Phys. Rev. Lett. 84 (2000) 4047; hep-ex/0104005.

[32] B.Q. Ma, I. Schmidt, and J.J. Yang, hep-ph/0110324, Phys. Rev. D (2002) to be published. 
[33] X. Artru and M. Mekhfi, Z. Phys. C 45 (1990) 669; Nucl. Phys. A 532 (1991) $351 \mathrm{c}$.

[34] M. Miyama and S. Kumano, Comput. Phys. Commun. 94 (1996) 185; M. Hirai, S. Kumano, and M. Miyama, ibid. 108 (1998) 38.

[35] DELPHI Collaboration, P. Abreu et al., Phys. Lett. B 318 (1993) 249.

[36] ALEPH Collaboration, D. Buskulic et al., Z. Phys. C 64 (1994) 361.

[37] G.D. Lafferty, P.I. Reeves, and M.R. Whalley, J. Phys. G 21 (1995) A1.

[38] OPAL Collaboration, G. Alexander et al., Z. Phys. C 73 (1997) 569.

[39] L3 Collaboration, M. Acciarri et al., Phys. Lett. B 407 (1997) 389.

[40] SLD Collaboration, K. Abe et al., Phys. Rev. D 59 (1999) 052001.

[41] CTEQ Collaboration, H.L. Lai et al., Eur. Phys. J. C 12 (2000) 375.

[42] B.Q. Ma, I. Schmidt, J. J. Yang, Phys. Rev. D 63 (2001) 037501; A.V. Efremov, K. Goeke, M.V. Polyakov, and D. Urbano, Phys. Lett. B 478 (2000) 94.

[43] R.D. Tangerman and P.J. Mulders, Phys. Rev. D 51 (1995) 3357;

D. Boer and R. Tangerman, Phys. Lett. B 381 (1996) 305;

D. Boer and P.J. Mulders, Phys. Rev. D 57 (1998) 5780;

D. Boer, R. Jakob, and P.J. Mulders, Phys. Lett. B 424 (1998) 143.

[44] M. Anselmino and F. Murgia, Phys. Lett. B 483 (2000) 74. 